\title{
Watercress and Water Quality: The Effect of Phenethyl Isothiocyanate on the Mating Behaviour of Gammarus pulex
}

\author{
Melanie J. Dixon and Peter J. Shaw \\ Centre for Environmental Sciences, Faculty of Engineering and the Environment, University of Southampton, Highfield, \\ Southampton SO17 1BJ, UK \\ Correspondence should be addressed to Peter J. Shaw, ps@soton.ac.uk
}

Received 23 December 2010; Accepted 8 March 2011

Academic Editor: Michelle Bloor

Copyright ( 92011 M. J. Dixon and P. J. Shaw. This is an open access article distributed under the Creative Commons Attribution License, which permits unrestricted use, distribution, and reproduction in any medium, provided the original work is properly cited.

\begin{abstract}
Watercress releases phenethyl isothiocyanate (PEITC) upon wounding as a defence against herbivores. PEITC levels released from watercress farms are elevated due to cropping, washing, and processing and are thought to lead to adverse effects on Gammarus pulex in chalk streams. This study elucidates the sublethal effect of PEITC on reproductive behaviour of G. pulex, employing ex situ tests to investigate the disruption of precopular pairing under conditions simulating in situ exposure. Mean time to separation of precopular pairs was $89 \pm 6$ minutes for watercress wash water ( $1 \mathrm{~g}$ watercress per litre water) and $81 \pm 15$ minutes for pure PEITC $(1 \mu \mathrm{L} / \mathrm{L})$. Re-exposure to watercress wash water to simulate the pulsed operation at a watercress farm did not alter behavioural response. The repeated interruption of reproductive behaviour under in situ conditions would impair long-term reproductive success and could explain in part low abundance of G. pulex downstream of watercress farms.
\end{abstract}

\section{Introduction}

England has the principal resource of chalk streams and rivers in Europe, many of which are designated conservation sites (e.g., Sites of Special Scientific Interest and Special Areas of Conservation) [1]. Chalk streams support an abundant and diverse macroinvertebrate community with many specialised and rare species, for example, the fine-lined mussel, Pisidium tenuilineatum, and the mayfly Paraleptophlebia wemeri [2]. Gammarus spp. have been identified as keystone species [3] with the potential to exert disproportionately powerful effects on the community structure and ecosystem processes. Gammaridae, which usually exist in chalk streams in very large numbers, are the principal detritivore and dominate the prey assemblage. As generalists, they are found along the length of the river.

Although chalk streams favour a rich and diverse ecology [1], their use by humans imposes a risk to some biota. Chalk headwaters provide an ideal location for cultivation of watercress (Nasturtium officinale) as the nutrient content is naturally high and the constant temperature of aquiferderived water provides protection from winter frosts and promotes vegetation growth during the colder months [4]. Whilst watercress occurs naturally as a common macrophyte in most reaches of chalk streams and can dominate during the summer period [5], it has the capacity to release substances that exert toxicological impacts on freshwater organisms. In particular, watercress is a source of phenethyl isothiocyanate (PEITC), a compound derived from the catabolism of glucosinolates present in cell vacuoles within tissues of plants containing them [6]. Glucosinolates occur naturally in watercress and other Brassicaceae, many of which are important economic food crops. The primary hydrolysis product of the glucosinolate present in greatest quantities in watercress (i.e., 2-phenethyl glucosinolate, also known as gluconasturtiin) is 2-phenethyl isothiocyanate (PEITC). A number of studies identify the role of the degradation products in the defence of the plant against herbivorous insects [7-9]; freshwater systems possess few specialist herbivores, and chemical feeding deterrents provide the most effective means of protection against generalist herbivores $[10,11]$.

Watercress is chemically defended from generalist herbivory by the glucosinolate-myrosinase system and the 
PEITC thereby released upon attack. Isothiocyanates, in particular PEITC, released by watercress have well-documented allelopathic and genotoxic properties [11] and have a role in the plant defence against herbivorous macroinvertebrates such as snails, caddis flies, and gammarids [12]. Few studies, however, have elucidated the effect of isothiocyanates on aquatic macroinvertebrates. The effectiveness of PEITC as a feeding deterrent has been established [13], and behavioural tests $[8,13,14]$ have shown avoidance of watercress by Gammarus pulex.

Despite these known impacts of PEITC on G. pulex, both watercress and $G$. pulex are considered characteristic of chalk streams [1] and are often observed at high abundance [4]. In some cases, however, low numbers of G. pulex have been linked to watercress farming and processing operations. For example, a reach of the Bourne Rivulet (Hampshire, UK; a tributary of the River Test) is entirely maintained by waters discharged from a watercress farm and washing and packing operation. Only watercress is grown at this site, but the washing and packing operation handles a variety of salad leaf products, including other Brassicaceae. Bruising, maceration, and wounding of watercress and other produce during harvesting and processing triggers the glucosinolatemyrosinase system, thereby increasing PEITC levels in the discharge waters. In this specific case, the observed reduction in G. pulex numbers and species diversity has been of concern due to the status of the watercourse as a chalk stream headwater which has an important role in the functioning of the River Test ecosystem downstream [15]. Measurement of PEITC from an aqueous matrix is, however, problematic and no standard methodology has been established.

For this particular location, the depleted abundance of G. pulex [15] may be due to the stream containing elevated concentrations of PEITC as a result of the entire stream being maintained by waters used in the watercress farm and washing and packing operation. There is clearly a need to improve our understanding of the causes of low $G$. pulex numbers in streams affected by watercress production and processing, such that effective mitigation measures may be informed by a quantitative scientific evidence base. We suggest that the low abundance of G. pulex could be explained by two ecotoxicological responses to PEITC. First, there may be an acute effect whereby mortality is substantial but incomplete. Second, there may be a sublethal effect whereby reproduction is impaired. Acute and sublethal effects are not mutually exclusive; this study forms part of a series of works and will focus on sublethal effects on reproduction in this instance.

G. pulex undertake a period of mate-guarding behaviour prior to mating. An adult male takes hold of a female and the pair remains together in precopular position for a few days until the female moults. Mating then occurs before her cuticle hardens, and the fertilised eggs are laid into a brood pouch. The female becomes attractive to males again at, or slightly before, the hatching of the eggs [16]. Precopular pairing may be affected by exposure to toxicants and separation has been reported as a sensitive and rapid indicator of stress due to cadmium [17] and raised ammonia levels [18]. Precopular separation along with neonatal and population growth are reported as consistently sensitive endpoints in an evaluation of methods used to evaluate toxicity to freshwater ecosystems [19]. The reproductive behaviour test has also been used to determine the effects of vertebrate-type endocrine disrupting chemicals; there is also evidence to support the use of pheromonal control of mating in G. pulex [20].

This overall aim of this study, therefore, is to determine the extent of the effects of PEITC on precopular pairing of $G$. pulex. We note that in situ exposure of G. pulex to substances derived from the watercress farm and processing operation occurs on a pulsed basis. The large area of watercress cropping beds and the crop washing facility onsite operates primarily on an "office hours" basis leading to potentially elevated levels of PEITC arising on a 24-hour cycle. Specifically, this study aims to (1) quantify the effects of exposure to PEITC on separation of precopular G. pulex pairs, (2) determine the effects of re-exposure on precopular separation, and (3) assess whether exposure impairs reformation of precopular pairs.

\section{Materials and Methods}

2.1. Preparation of Test Solutions. A single batch of mature (i.e., ready for harvest) watercress was washed briefly to remove coarse debris. Care was taken to minimise handling damage to avoid subsequent loss of PEITC from the sample. The sample was then frozen to store for tests and prevent further hydrolysis of glucosinolate to PEITC. A single batch was used to minimise variability in the glucosinolate concentration in crops grown under different conditions $[21,22]$. Media (dilution) water was prepared by vigorously aerating tap water for more than two hours to remove chlorine. Frozen watercress leaves and stems (large stems were excluded) were weighed and added to a measured volume of media water. The media water/leaf mixture was stirred once, that is, a stirring rod making one revolution of the beaker and then leaf and stem debris was filtered out using a $250 \mu \mathrm{m}$ mesh. The resulting wash water was used as the test solution. It was assumed that the freezing process had caused complete lysis of cell walls and thus complete and immediate hydrolysis of glucosinolate to PEITC. Analytical grade PEITC was used to prepare PEITC test solutions. PEITC is heat and moisture sensitive [23] and required dilution with analytical grade methanol in a stock solution. Test solutions were made on the day of the test $(1 \mu \mathrm{L} / \mathrm{L})$ by dilution of the stock with aerated media water.

2.2. Test Organisms. G. pulex were collected from the River Meon at Funtley Mill, Hampshire (NGR SU556089). They were acclimatised to laboratory conditions in a constant temperature room at $14 \pm 2^{\circ} \mathrm{C}$, with a photoperiod of 8 hours daylight and 16 hours darkness under cool white fluorescent tubes (mean bench-top illumination of 800 lux), in glass tanks with tap water media which had been vigorously aerated for more than two hours to remove all chlorine. They were fed a diet of alder leaves (Alnus glutinosa (L.)) presoaked in river water, and $10 \%$ (by volume) media changes were made every two days for a period of two weeks. 
The breeding population was then maintained under these conditions.

Precopular pairs were used for sublethal tests as the interruption of reproductive behaviour would be indicative of an unsustainable population. The use of sublethal data would also provide a greater level of sensitivity and in applying the results to the process at the study site would afford a greater degree of protection within the receiving water. Initial trials resulted in immediate separation of control precopular pairs due to handling stress when they were transferred to and from the holding vessel media; several methods employ physical stimulation as a technique to isolate males from females [24-26]. Of a number of different methods examined for the transfer of precopular pairs (e.g., use of a wide bore pipette, a sieve, a spoon, or emptying out media), the advantage due to minimising handling stress was compromised by other factors such as the time taken or the potential dilution of the test solution by media water. The use of the wide bore pipette was thus chosen, providing minimal handling stress and transfer of media water but not impractically prolonging the transfer of organisms to the test solution.

2.3. Quality Control. Tests were carried out as far as possible according to quality control methods prescribed by laboratory standard ISO 17025 [27]. Daily temperature checks were carried out to ensure the constant temperature room remained within an acceptable temperature range. Equipment used was calibrated using United Kingdom Accreditation Service (UKAS) approved methods (e.g., balance, Finn-pipettes, water quality meters, and timers). Calibrated volumetric glassware was used. Solvent controls (analytical grade methanol diluted to the same concentration as in the PEITC test solution with aerated media water) and media controls were carried out for tests using PEITC test solutions and media controls carried out for tests using watercress wash water test solutions. All control organisms were subject to the same handling as the test organisms. Water quality validation criteria for dissolved oxygen $(>60 \%$ ASV), $\mathrm{pH}$ (constant to within 0.5 unit), conductivity $(<10 \%$ change) were also assessed for each test. No adjustment or correction of test solutions was required as validity criteria were met on all occasions.

2.4. Two-Hour Time to Pair-Separation Test. As part of their mating behavior, G. pulex form precopulatory pairs, separating once fertilisation has taken place $[20,28]$. Initial observations of precopulatory pairs in wash water were made with a view to carrying out the precopulatory separation (GaPPs) test described by Pascoe et al. [28]. This procedure exposes pairs to the test solution for one hour followed by an enforced separation and records the time taken for pairs to reform. However, watercress wash water caused pair separation during the one-hour exposure and a variation of this method was used in which precopulatory pairs were exposed to a single dose of watercress wash water for a two-hour period. The concentration of watercress wash water test solution selected was guided by the ratio of leaf to water washed in the salad washing and processing factory, which washes at a ratio of $1 \mathrm{~g}$ leaf to $50 \mathrm{~mL}$ water [29]. Isothiocyanate-producing crops make up approximately $50 \%$ of product washed; accordingly, a concentration equivalent to $1 \mathrm{~g}$ watercress per $100 \mathrm{~mL}$ wash water was used. The endpoint used was time to separation of pairs and was recorded at 15-minute intervals. Glass crystallising dishes covered with a watch glass were used as the test vessel, with $150 \mathrm{~mL}$ of test solution and 5 precopular pairs added to each test vessel. At least 4 (and up to 8 ) replicate vessels were employed, depending on the number of pairs available.

2.5. Precopular Re-Exposure Test. A series of re-exposure tests was also conducted to elucidate responses of precopular pairs to pulsed exposures as experienced in the Bourne Rivulet. The wash process at the farm operates daily $(0730$ to $1700 \mathrm{~h}$ weekdays and 0630 to $1600 \mathrm{~h}$ weekends) and outside this the discharge consists of borehole water from watercress bed flow only. Consequently, there is a period every 24 hours when there may be very low levels of PEITC present in the discharge. During the processing hours, the wash lines are changed at frequent intervals, for example, on 10 June 2008, there were 43 different product lines washed and packaged, and each product contained a varying proportion of watercress in the total weight washed [30]. The nature of the discharge from the processing operation is thus highly variable. Re-exposures were carried out in a laboratory simulation of the temporally variable nature of the wash and process factory water, but variation of the crop processed was not simulated. At the end of the two-hour precopular separation test, the test organisms were removed to clean water and left to re-pair over a period of $48 \mathrm{hrs}$. The re-paired organisms were then re-exposed to fresh test solution as per the first test.

\section{Results}

3.1. Sublethal Tests. Four tests were carried out with watercress wash water as the test solution. Test organisms from two of these were re-exposed to freshly prepared watercress wash water, one at test end plus 24 hours and the other at test end plus 48 hours. Similarly, four tests were carried out using a PEITC test solution and test organisms from two of these were re-exposed, one at test end plus 24 hours and the other at test end plus 48 hours. Both the watercress wash water and the PEITC solution disrupted reproductive behaviour (Figure 1). The $\mathrm{ET}_{50}$ (i.e., the exposure duration at which $50 \%$ of precopular pairs had their natural behaviour disturbed and separated) was calculated by hypothesis testing for each test using ToxCalc v5.0.32 environmental toxicity data analysis software [31]. Calculated $\mathrm{ET}_{50}$ values (Table 1) varied markedly between tests, but with considerable overlap of the ranges for tests using watercress wash water and a solution of PEITC, as also demonstrated by separation patterns over the whole two-hour period (Figure 1).

3.2. Re-Exposures. On re-exposure to freshly prepared watercress wash water and PEITC solution at the same concentration as the first exposure, pair separation was observed in a 


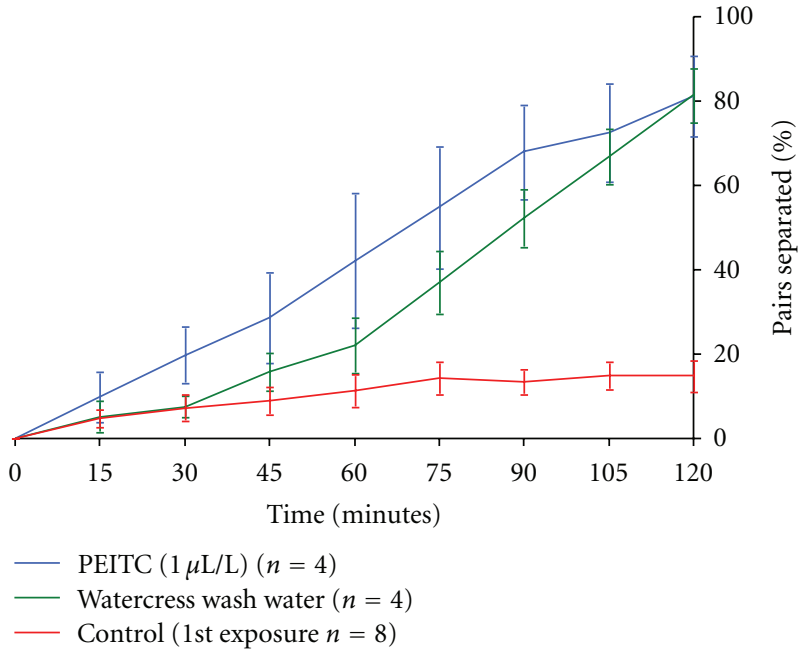

Figure 1: Mean cumulative proportion of pairs separated $( \pm \mathrm{SE})$. Precopular pairs were exposed at Time $=0$, the mean of 4 tests over the course of the $2 \mathrm{~h}$ exposure is shown for each test substance (carried out on separate occasions, with a total of 119 pairs exposed to wash water, 105 pairs exposed to PEITC) and the control (mean of 8 tests, with a total of 157 pairs exposed). Vertical bars show standard error; $n$ denotes the number of tests. There was immediate separation of at least one pair in all the PEITC test solutions (i.e., by the first 15-minute reading). There was separation of at least one pair in all the watercress wash water test solutions after 45 minutes. There was a steady increase in the number of pairs separated over the course of the two-hour test to $70 \%$ or greater in all wash water test solutions (maximum 95\%, mean 84\%) at the test end. The pattern of response for the PEITC solution was very similar (maximum $100 \%$, mean $85 \%$ ) at test end.

TABle 1: Summary of $\mathrm{ET}_{50}$ values. The proportional data were arcsine square root transformed and the $\mathrm{ET}_{50}$ calculated using maximum-likelihood probit or logit analysis. WW1-WW5: samples of watercress wash water. P1-P5: samples of PEITC solution.

\begin{tabular}{lcc}
\hline Sample & $\mathrm{ET}_{50}$ (minutes) & $95 \%$ confidence intervals \\
\hline WW1 & $77^{*}$ & $73-85$ \\
WW2 & $106^{*}$ & $103-110$ \\
WW3 & 89 & $78-102$ \\
WW5 & 84 & $72-93$ \\
P1 & 48 & $38-56$ \\
P2 & 119 & $108-133$ \\
P3 & 85 & $77-92$ \\
P5 & 40 & $20-56$ \\
\hline
\end{tabular}

${ }^{*}$ Calculated using logit model—all others with probit.

similar manner as for the first exposure; however, it occurred sooner (Figures 2 and 3) and resulted in an overall greater proportion separation after two hours (Figure 4) although there was no statistically significant difference. The $\mathrm{ET}_{50}$ (95\% CI) values for pairs re-exposed to watercress wash

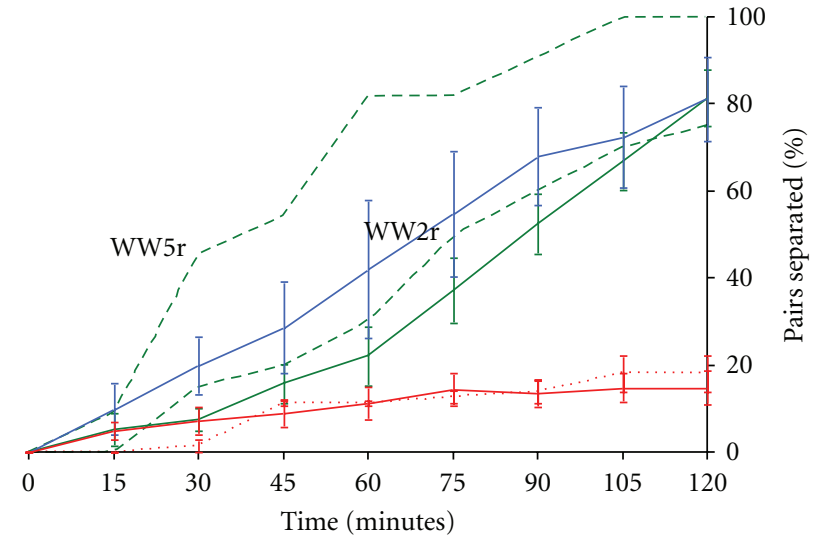

$\begin{array}{ll}- & \text { Wash water }(n=4) \quad-- \text { Watercress re-exposure } \\ - & \text { PEITC }(1 \mu \mathrm{L} / \mathrm{L})(n=4) \quad-\text { Control }(n=8) \\ \ldots . . . & \text { Control re-exposures }(n=4)\end{array}$

FIgURE 2: Cumulative proportion of pairs separated-watercress wash water re-exposures. Solid lines show the mean response for four separate initial exposures. The initial wash water exposure (green) is emboldened for comparison with re-exposures (greendotted lines) to wash water on 2 separate test occasions; Test WW5r (11 pairs) after exposed test organisms had spent $24 \mathrm{~h}$ in clean water, and Test WW2r (20 pairs) after exposed test organisms had spent $48 \mathrm{~h}$ in clean water. The mean response of 4 control re-exposures (red) follows a similar pattern to the initial control exposure (reddotted line shows the mean of 8 control exposures). Vertical bars show standard error; $n$ denotes the number of tests.

water were 87 (77-106) and $41(27-51)$ minutes. The $\mathrm{ET}_{50}$ ( $95 \%$ CI) values for pairs re-exposed to PEITC solution were 54 (41-64) and 40 (19-53) minutes. For all re-exposures, the $\mathrm{ET}_{50}$ was reduced, that is, pair separation occurred sooner (Figure 5), although it should be noted that as only two reexposures were carried out, a statistically robust assessment of the variability could not be made. The rate of pairs reforming was assessed for organisms returned to clean water at the initial test end (Figure 6). In all instances except one (Test WW3), where the proportion of pairs re-forming was recorded, the number of pairs was greater after a period in clean water than at the end of the test exposure. After return to clean water, there was generally a proportion of G. pulex that did not re-pair and the mean control pair re-formation achieved was $75 \%(n=6)$. However, on a single occasion (test WW5), control re-formation was 100\%; the two control pairs that had separated during the initial test were able to reform in the following 24-hour period.

3.3. PEITC Concentration in Wash Water. Although analysis of the test solutions for PEITC was not carried out, GC-MS analyses of watercress wash water may give an indication of the amount of PEITC that the test organisms were exposed to. Dixon [32] estimated the amount of PEITC released by weight as a mean value of $529 \pm 45 \mu \mathrm{g} / \mathrm{g}$ leaf washed. Therefore, precopular pairs were exposed to PEITC at an estimated concentration of $5.3 \pm 0.5 \mathrm{mg} / \mathrm{L}$ PEITC. 


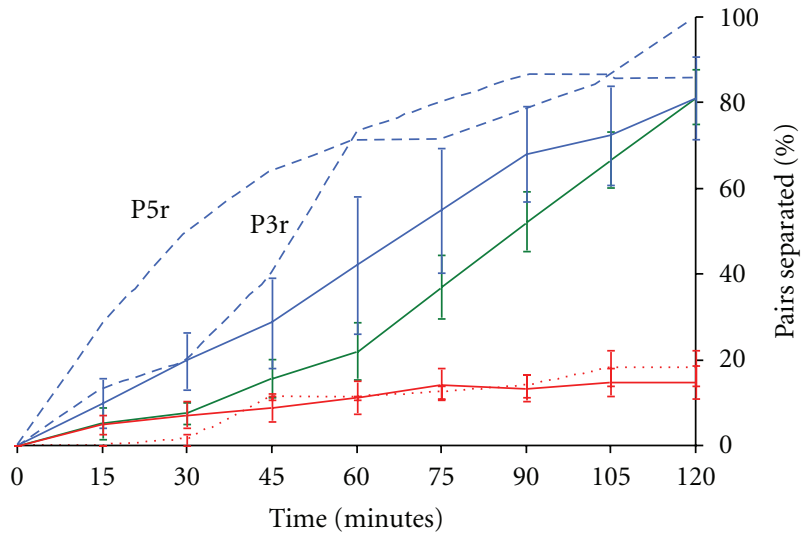

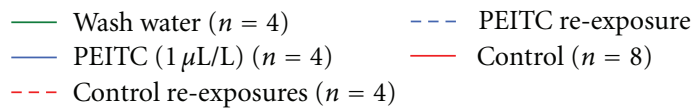

Figure 3: Cumulative proportion of pairs separated-PEITC reexposures. Solid lines show the mean response for four separate initial exposures. The initial PEITC exposure (blue) is emboldened for comparison with re-exposures (blue-dotted lines) to PEITC on 2 separate test occasions; Test P5r (14 pairs) after exposed test organisms had spent $24 \mathrm{~h}$ in clean water, and Test $\mathrm{P} 3 \mathrm{r}$ (15 pairs) after exposed test organisms had spent $48 \mathrm{~h}$ in clean water. The mean response of 4 control re-exposures (red) follows a similar pattern to the initial control exposure (red-dotted line shows the mean of 8 control exposures). Vertical bars show standard error; $n$ denotes the number of tests.

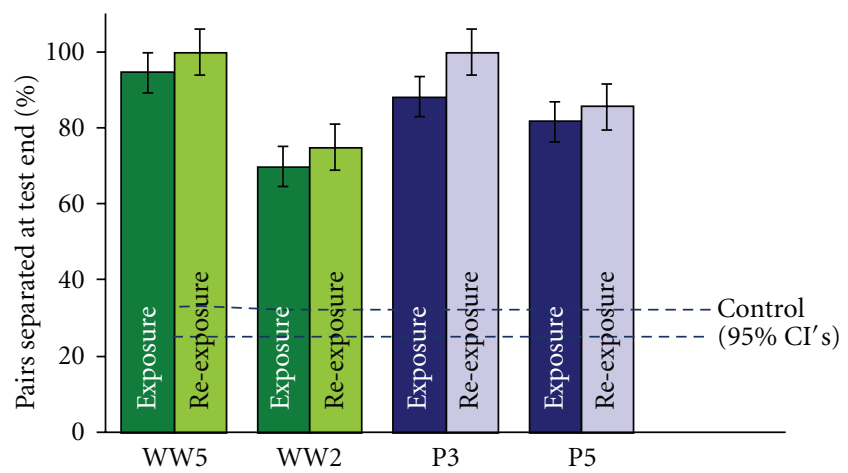

FIGURE 4: Proportion of pairs separated at two-hour test end $( \pm \mathrm{SE})$. Wash water exposures (WW5 and WW2) are shown as green, and PEITC exposures (P3 and P5) are shown as blue. The 95\% upper and lower confidence intervals for the initial control exposures are shown as dotted lines for comparative purposes.

\section{Discussion}

4.1. Sensitivity of Gammarus pulex to PEITC and Watercress Wash Water. The use of Gammarus spp. for ecotoxicological testing at both acute and sublethal levels of sensitivity has been well documented and evaluated [33-35] and includes the specific use of a precopular separation test [28]. Protocols for acute testing with G. fasciatus, G. pseudolimnaeus, and
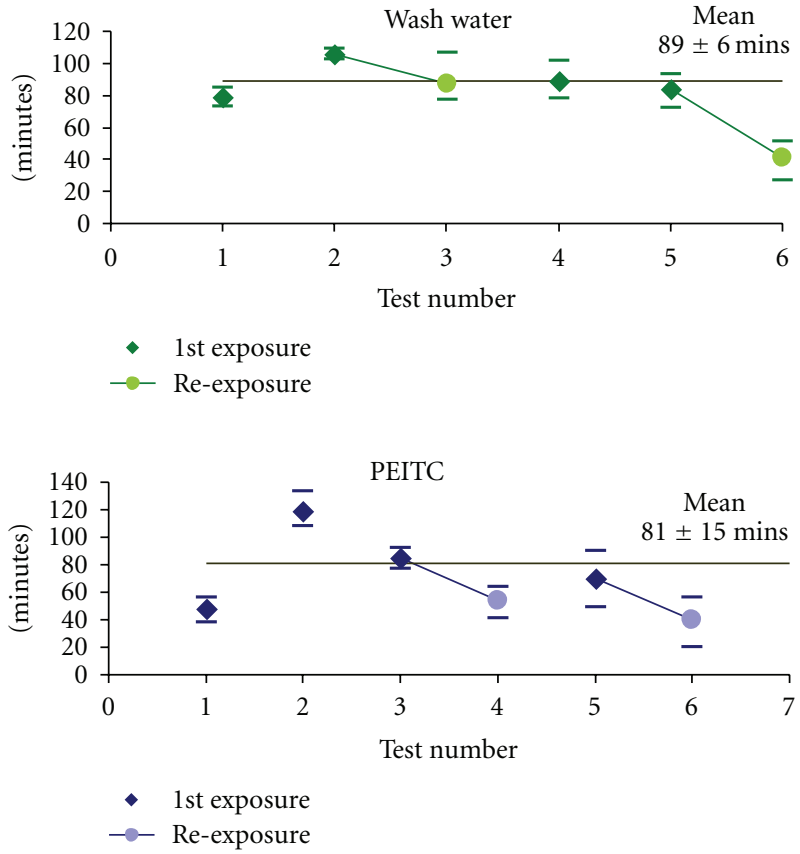

Figure 5: $\mathrm{ET}_{50}(95 \% \mathrm{CI})$ values for exposures and re-exposures. The upper panel shows the $\mathrm{ET}_{50}$ value for each PEITC exposure (green diamond) and re-exposure (green circle). The lower panel shows $\mathrm{ET}_{50}$ value for each wash water exposure (blue diamond) and re-exposure (blue circle). Re-exposures are linked to the initial exposure by a solid line. Horizontal bars show the 95\% upper and lower confidence intervals. Mean $\mathrm{ET}_{50}( \pm \mathrm{SE})$ value for the initial exposures is shown as a solid black line.

G. lacustris are available within the United States Environmental Protection Association test methods collection [36]. Johnson et al. [37] recognised the importance of appropriate bioassay choice, design, and quality assurance/quality control measures in effluent assessment and control. The choice of $G$. pulex as the test organism in this study was influenced by the observed impact on Gammaridae recorded in the receiving water downstream of the watercress farm and processing operation at the study site.

Application of sublethal tests as employed in the present study is influenced by the nature of PEITC. This compound has an unknown degradation pathway in watercress wash water, which may depend on temperature, $\mathrm{pH}$ [38], and/or the presence of other members of the family Cruciferae [39] and the volatility of glucosinolate breakdown products [6]. The volatility of PEITC constrains longer-term tests; a continual dosing system would not have been practicable for this study. It was possible to achieve a precopular separation endpoint over a short period of exposure to wash water solution and the response was also recorded throughout the duration of the two-hour exposure period. This response was similar for the watercress wash water solution, the reexposed organisms, and the PEITC solution although for the re-exposures occurred sooner.

The mode of action of PEITC from watercress wash water has not yet been established, although many studies have documented the relationship between terrestrial herbivorous 


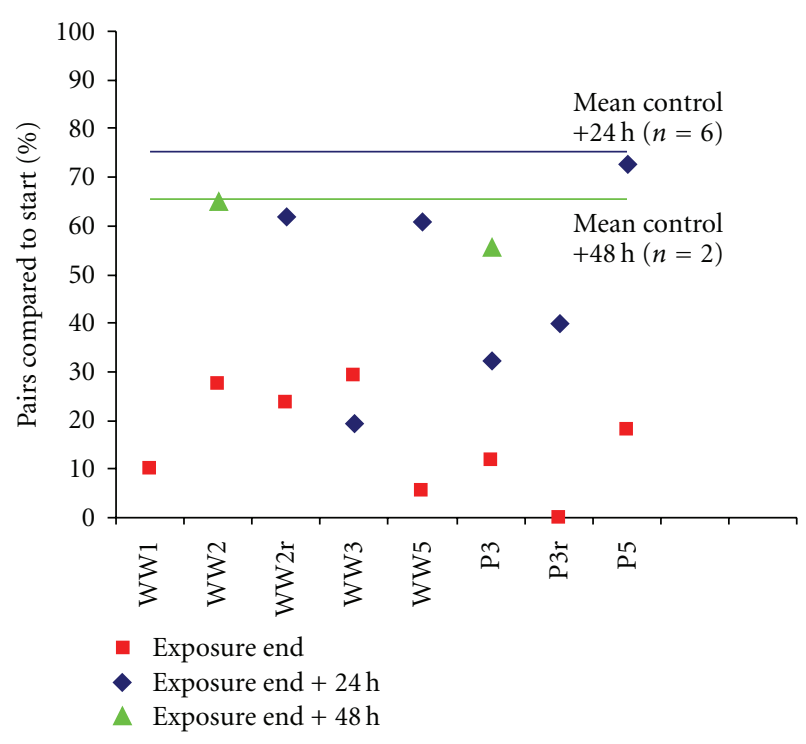

Figure 6: The proportion of pairs re-forming after their return to clean water at exposure end. The proportion of pairs re-forming after transfer to clean water at the initial exposure test end is shown for each separate test occasion. The proportion of pairs present at the start of the first exposure was taken as $100 \%$. The mean control re-pairing is shown for comparison, after $24 \mathrm{~h}$ in clean water mean of 6 tests (blue line) and after $48 \mathrm{~h}$ in clean water mean of 2 tests (green line). Wash water exposures (WW); PEITC exposures (P); proportion of pairs remaining at initial exposure end (red square); after $24 \mathrm{~h}$ in clean water (green triangle); after $48 \mathrm{~h}$ in clean water (blue diamond). NB: Re-pairing was not recorded after test WW1. $n$ denotes the number of tests.

invertebrates and glucosinolate producing crops [40-43] and the use of chemoreceptors in adaptive behaviour. Watercress wash water has elicited a response in adults [40-44], feeding adults [8], and reproductive adults (this study). Therefore, although the ingestion of PEITC may cause an acute response, it is possible that detection of PEITC by chemoreceptors or its metabolism within cells may also be eliciting the sublethal behaviour that has been recorded. In studies relating to the use of PEITC as an anticarcinogen, which have investigated and quantified the uptake of isothiocyanates, rapid cellular uptake has been demonstrated [45-47]. Rapid cellular uptake would concur with the sublethal response by reproductive G. pulex, seen within 2 hours, and their subsequent recovery. We therefore speculate that the mode of toxic action of PEITC on Gammarus spp. is probably initially at a cellular level. PEITC may additionally act separately via ingestion [8] with long-term exposure (exposure possibly via several pathways) leading to mortality.

The effect of re-exposing precopular pairs to watercress wash water and PEITC solution was analysed using four different methods. The graphical comparison of rates of separation during the two-hour exposure (Figures 2 and 3 ) illustrated that the effect was seen more quickly in organisms already exposed to the toxicant. This result was supported with lower $\mathrm{ET}_{50}$ values for exposures to both watercress wash water and PEITC than for the initial exposures, that is, the effect would be seen in half of the population more quickly than for the first exposure. The two-hour proportion separated showed that the overall sensitivity of the preexposed organisms was however not significantly increased.

4.2. Practical Implications. Exposure to watercress wash water and PEITC produced a behavioural response measurable in reproductive adults. The behavioural response seen in reproductive adults was carried out at a single concentration and it is not clear from this work whether fluctuations in their response would be altered by a change in the dose regimen. It is possible that with an increase in the exposure duration and/or if the dose was increased beyond a certain level, the separation of reproductive pairs may give way to a toxic response leading to adult mortality. There will, therefore, be implications for the sustainability or survival of populations of $G$. pulex in the receiving water below watercress farm discharges where exposure to PEITC leads to similar doses to those used in this study.

The reversibility of the behavioural response may also depend on the exposure duration and dose. Returning organisms to fresh water at test end allowed the interrupted reproductive behaviour to recommence; at the dose tested, the separation was due to a transient effect. However, it is important to note that the opportunity for male G. pulex to fertilise females is limited to a few hours after the female moults [16]. The mate guarding behaviour thus ensures access to the female when she is receptive. In relation to operations at the study site, the repeated disruption by daily pulses of discharge of watercress wash water would reduce the opportunities for males to fertilise and therefore, over a long period, reduce the reproductive success of the population.

Analysis of the number of pairs re-forming showed that there was an inconsistent increase in pair re-forming over a 48 -hour period, and even in controls, $100 \%$ re-pairing was not generally achievable. The number of pairs re-forming was also subject to the natural pattern of the reproductive cycle [16], and thus a proportion would naturally separate anyway. It is interesting to note that separation of re-exposed pairs (Figures 2 and 3 ) occurred sooner in the tests which were carried out after $24 \mathrm{~h}$ rather than $48 \mathrm{~h}$ in clean water, even though this was not reflected in an overall greater proportion separation at the end of the two-hour period or a much lower $\mathrm{ET}_{50}$.

Where low diversity or low abundance is noted in the macroinvertebrate populations of chalk stream receiving waters below watercress farms, the potential effects due to PEITC should be considered. Watercress producers are required to meet consent conditions for a variety of water quality parameters such as suspended solid load and biological oxygen demand (BOD). The contribution of PEITCinduced effects should also be examined.

4.3. Wash Water Sample Preparation. The method of preparation of watercress wash water test solution was based on the salad wash process at the study site. Sources of variation were minimised, particularly where nominal concentrations 
were prepared for the acute test using very small quantities of leaf only. Wash water prepared using larger quantities of both leaf and stem may have introduced variability due to the potential for different glucosinolate content in each part of the plant. Although a comparison of PEITC present in stem and leaves has not been made, Gil and Macleod [39] showed that there were different levels of PEITC produced from $N$. officinale seeds and leaves. Likewise, Rosa [48] described significant variation between glucosinolates present in the roots and aerial parts of Brassica seedlings. Newman et al. [14] also reported that toxicity of frozen watercress roots to G. pseudolimnnaeus was similar to the leaves.

4.4. Further Work. It was only possible to carry out reexposure tests when there were sufficient precopulatory pairs after the return of test organisms to clean water. The use of much larger numbers of pairs in the initial tests would have resulted in more pairs becoming available for reexposure. However, this was governed on a practical basis by the facilities and human resources available for test setup. Similarly, a longer time period in clean water may have increased the numbers of pairs available for re-exposure. A compromise was made between practicability and relevance to field simulation at the farm where re-exposures occur within 24 hours. Re-exposure tests were carried out where at least 3 replicates of 3 pairs were possible as well as control replicates, although this was less than recommended by standardised acute test methodology such as Environment Agency [49] acute single concentration Daphnia magna test where 6 replicates and 20 organisms are prescribed. It should be recognised that the use of a larger number of pairs would have increased the statistical robustness of the method.

Further testing with freshly collected samples of salad wash water, taken directly from the wash lines at the study site, would provide a direct link to the crop washing process and its effect on gammarids in the receiving water. It would additionally be beneficial to increase the number of sublethal tests carried out with PEITC solution and watercress wash water to assess the level of variability in the G. pulex response and confirm the reproducibility of the test. The reliability of the short-term sublethal test could also be evaluated by further tests to establish the natural background variability against which the stress-induced precopular separation can be measured [33]. An estimate of the unimpaired repairing rate for the population could be made by artificially separating control organisms prior to a period in clean water.

\section{Conclusions}

The secondary metabolite PEITC produced by harvested and processed watercress has a sublethal effect on G. pulex breeding pairs. The effect is evident at concentrations anticipated to be produced by the leaf washing process at the study site. Re-exposures of G. pulex precopular pairs to PEITC in watercress leaf wash water did not illicit a significantly different separation response although all re-exposures had a lower $\mathrm{ET}_{50}$ and responded more quickly during the exposure. The organisms did not appear to acclimatise to PEITC or become less able to withstand its effect. Further tests and reexposures would establish if this was a consistent finding. The adaption and extension of a more commonly used reproductive pair separation methodology (i.e., to re-expose organisms to freshly prepared test solution) reflected more accurately the exposure pattern experienced by organisms in the receiving environment. This novel use was considered important to the relevance of the particular situation in the receiving water below the discharge from the study site. The mode of action of the toxicant has not been confirmed, although behavioural effects are evident. The similar response seen in both PEITC solution and watercress leaf wash water solution would indicate that PEITC is the causative agent.

\section{Acknowledgment}

The authors gratefully acknowledge the financial support of this study by the Vitacress Conservation Trust.

\section{References}

[1] Environment Agency, The State of England's Chalk Rivers, UK Biodiversity Action Plan Steering Group for Chalk Rivers Environment Agency, Bristol, UK, 2004.

[2] Hampshire Biodiversity Partnership, Biodiversity Action Plan for Hampshire, vol. 2, Hampshire County Council, 2000.

[3] G. Woodward, G. Papantoniou, F. Edwards, and R. B. Lauridsen, "Trophic trickles and cascades in a complex food web: impacts of a keystone predator on stream community structure and ecosystem processes," Oikos, vol. 117, no. 5, pp. 683-692, 2008.

[4] A. D. Berrie, "The chalk-stream environment," Hydrobiologia, vol. 248, no. 1, pp. 3-9, 1992.

[5] C. P. Mainstone, Chalk Rivers Nature Conservation and Management, WRc, 1999.

[6] A. M. Bones and J. T. Rossiter, "The myrosinase-glucosinolate system, its organisation and biochemistry," Physiologia Plantarum, vol. 97, no. 1, pp. 194-208, 1996.

[7] J. W. Fahey, A. T. Zalcmann, and P. Talalay, "The chemical diversity and distribution of glucosinolates and isothiocyanates among plants," Phytochemistry, vol. 56, no. 1, pp. 5$51,2001$.

[8] R. M. Newman, Z. Hanscom, and W. C. Kerfoot, "The watercress glucosinolate-myrosinase system: a feeding deterrent to caddisflies, snails and amphipods," Oecologia, vol. 92, no. 1, pp. 1-7, 1992.

[9] W. C. Kerfoot, R. M. Newman, and Z. Hanscom, "Snail reaction to watercress leaf tissues: reinterpretation of a mutualistic "alarm" hypothesis," Freshwater Biology, vol. 40, no. 2, pp. 201-213, 1998.

[10] A. C. Prusak, J. O'Neal, and J. Kubanek, "Prevalence of chemical defenses among freshwater plants," Journal of Chemical Ecology, vol. 31, no. 5, pp. 1145-1160, 2005.

[11] A. L. Shelton, "Within-plant variation in glucosinolate concentrations of Raphanus sativus across multiple scales," Journal of Chemical Ecology, vol. 31, no. 8, pp. 1711-1732, 2005.

[12] J. Brown and M. J. Morra, Glucosinolate-Containing Seed Meal as a Soil Amendment to Control Plant Pests: 2000-2002, National Renewable Energy Laboratory, 2005.

[13] R. M. Newman, W. C. Kerfoot, and Z. Hanscom, "Watercress allelochemical defends high-nitrogen foliage against 
consumption: effects on freshwater invertebrate herbivores," Ecology, vol. 77, no. 8, pp. 2312-2323, 1996.

[14] R. M. Newman, W. C. Kerfoot, and Z. Hanscom, "Watercress and amphipods: Potential chemical defense in a spring stream macrophyte," Journal of Chemical Ecology, vol. 16, no. 1, pp. 245-259, 1990.

[15] S. Medgett, The Impact of St Mary Bourne Cress Farm on the Bourne Rivulet, Ecological Appraisal Team, Hampshire and Isle of Wight Area Environment Agency, 1998.

[16] H. B. N. Hynes, "The reproductive cycle of some British freshwater Gammaridae," The Journal of Animal Ecology, vol. 24, no. 2, pp. 352-387, 1955.

[17] C. P. McCahon and D. Pascoe, "Use of Gammarus pulex (L.) in safety evaluation tests: culture and selection of a sensitive life stage," Ecotoxicology and Environmental Safety, vol. 15, no. 3, pp. 245-252, 1988.

[18] J. Prenter, C. MacNeil, J. T. A. Dick, G. E. Riddell, and A. M. Dunn, "Lethal and sublethal toxicity of ammonia to native, invasive, and parasitised freshwater amphipods," Water Research, vol. 38, no. 12, pp. 2847-2850, 2004.

[19] A. E. Girling, D. Pascoe, C. R. Janssen et al., "Development of methods for evaluating toxicity to freshwater ecosystems," Ecotoxicology and Environmental Safety, vol. 45, no. 2, pp. 148 $176,2000$.

[20] M. M. Watts, D. Pascoe, and K. Carroll, "Survival and precopulatory behaviour of Gammarus pulex (L.) exposed to two xenoestrogens," Water Research, vol. 35, no. 10, pp. 23472352, 2001

[21] G. Engelen-Eigles, G. Holden, J. D. Cohen, and G. Gardner, "The effect of temperature, photoperiod, and light quality on gluconasturtiin concentration in watercress (Nasturtium officinale R. Br.)," Journal of Agricultural and Food Chemistry, vol. 54, no. 2, pp. 328-334, 2006.

[22] U. Palaniswamy, R. McAvoy, and B. Bible, "Supplemental light before harvest increases phenethyl isothiocyanate in watercress under 8-hour photoperiod," HortScience, vol. 32, no. 2, pp. 117-205, 1997.

[23] Sigma-Aldrich, "Safety Data Sheet: phenethyl isothiocyanate 253731 (Version 3.0, Revision Date 28/8/09), " in According to Regulation (EC) 1907/2006, 2009.

[24] A. Cold and V. E. Forbes, "Consequences of a short pulse of pesticide exposure for survival and reproduction of Gammarus pulex," Aquatic Toxicology, vol. 67, no. 3, pp. 287-299, 2004.

[25] J. F. C. Malbouisson, T. W. K. Young, and A. W. Bark, "Disruption of precopula in Gammarus pulex as a result of brief exposure to gamma-hexachlorocyclohexane (Lindane)," Chemosphere, vol. 28, no. 11, pp. 2011-2020, 1994.

[26] E. W. Sexton, "On the rearing and breeding of Gammarus in laboratory conditions," Journal of the Marine Biological Association of the United Kingdom, vol. 15, no. 1, pp. 33-56, 1928.

[27] International Organisation for Standardisation, General requirements for the competence of testing and calibration laboratories, 2nd edition, 2005.

[28] D. Pascoe, T. J. Kedwards, S. J. Maund, E. Muthi, and E. J. Taylor, "Laboratory and field evaluation of a behavioural bioassay: the Gammarus pulex (L.) precopula separation (GaPPS) test," Water Research, vol. 28, no. 2, pp. 369-372, 1994.

[29] Vitacress Salads Ltd, Estimate of Watercress: Wash Water Ratio, Lower Link Farm, E. Hiscocks, 2008.

[30] Vitacress Salads Ltd, Crop Wash Statistics May-June 2007 \& June-July 2008, M. Fisher, 2008.
[31] Tidepool Scientific Software, ToxCalc: Environmental Toxicity Data Analysis System Version 5.0.32, McInleyville, Calif, USA, 1994.

[32] M. J. Dixon, The sustainable use of water to mitigate the impacts of watercress farms on chalk streams in Southern England, Ph.D. thesis, Department of Civil Engineering and the Environment, University of Southampton, 2010.

[33] L. Maltby, S. A. Clayton, R. M. Wood, and N. McLoughlin, "Evaluation of the Gammarus pulex in situ feeding assay as a biomonitor of water quality: robustness, responsiveness, and relevance," Environmental Toxicology and Chemistry, vol. 21, no. 2, pp. 361-368, 2002.

[34] E. J. Taylor, D. P. Jones, S. J. Maund, and D. Pascoe, "A new method for measuring the feeding activity of Gammarus pulex (L)," Chemosphere, vol. 26, no. 7, pp. 1375-1381, 1993.

[35] J. S. Welton and R. T. Clarke, "Laboratory studies on the reproduction and growth of the amphipod, Gammarus pulex (L.)," Animal Ecology, vol. 49, pp. 581-592, 1980.

[36] USEPA, "Gammarid acute toxicity test (OTS-795-120)," in EPA Test Methods, 1996.

[37] I. Johnson, M. Hutchings, R. Benstead, J. Thain, and P. Whitehouse, "Bioassay selection, experimental design and quality control/assurance for use in effluent assessment and control," Ecotoxicology, vol. 13, no. 5, pp. 437-447, 2004.

[38] Y. Ji, Y. Kuo, and M. E. Morris, "Pharmacokinetics of dietary phenethyl isothiocyanate in rats," Pharmaceutical Research, vol. 22, no. 10, pp. 1658-1666, 2005.

[39] V. Gil and A. J. MacLeod, "Degradation of glucosinolates of Nasturtium officinale seeds," Phytochemistry, vol. 19, no. 8, pp. 1657-1660, 1980.

[40] V. M. Koritsas, J. A. Lewis, and G. R. Fenwick, "Glucosinolate responses of oilseed rape, mustard and kale to mechanical wounding and infestation by cabbage stem flea beetle (Psylliodes chrysocephala)," Annals of Applied Biology, vol. 118, no. 1, pp. 209-221, 1991.

[41] P. W. Lambdon and M. Hassall, "Do plant toxins impose constraints on herbivores? An investigation using compartmental analysis," Oikos, vol. 93, no. 1, pp. 168-176, 2001.

[42] P. Roessingh, E. Städler, G. R. Fenwick et al., "Oviposition and tarsal chemoreceptors of the cabbage root fly are stimulated by glucosinolates and host plant extracts," Entomologia Experimentalis et Applicata, vol. 65, no. 3, pp. 267-282, 1992.

[43] K. Rowell and D. W. Blinn, "Herbivory on a chemically defended plant as a predation deterrent in Hyalella azteca," Freshwater Biology, vol. 48, no. 2, pp. 247-254, 2003.

[44] A. D. P. Worgan and R. Tyrell, "Monitoring behavioural responses of Gammarus pulex to watercress oils," Centre for Ecology and Hydrology, Unpublished Report to Vitacress Salads Ltd CEH Project no. 602786NEW, 2005.

[45] Y. Zhang, "Molecular mechanism of rapid cellular accumulation of anticarcinogenic isothiocyanates," Carcinogenesis, vol. 22, no. 3, pp. 425-431, 2001.

[46] F. L. Chung, M. A. Morse, K. I. Eklind, and J. Lewis, "Quantitation of human uptake of the anticarcinogen phenethyl isothiocyanate after a watercress meal," Cancer Epidemiology Biomarkers and Prevention, vol. 1, no. 5, pp. 383-388, 1992.

[47] J. W. Chiao, H. Wu, G. Ramaswamy et al., "Ingestion of an isothiocyanate metabolite from cruciferous vegetables inhibits growth of human prostate cancer cell xenografts by apoptosis and cell cycle arrest," Carcinogenesis, vol. 25, no. 8, pp. 14031408, 2004.

[48] E. A. S. Rosa, "Daily variation in glucosinolate concentrations in the leaves and roots of cabbage seedlings in two constant 
temperature regimes," Journal of the Science of Food and Agriculture, vol. 73, no. 3, pp. 364-368, 1997.

[49] Environment Agency, Methods for the examination of waters and associated materials: The Direct Toxicity Assessment of aqueous environmental samples using the juvenile Daphnia magna immobilisation test, Standing Committee of Analysts, 2007. 



Submit your manuscripts at

http://www.hindawi.com
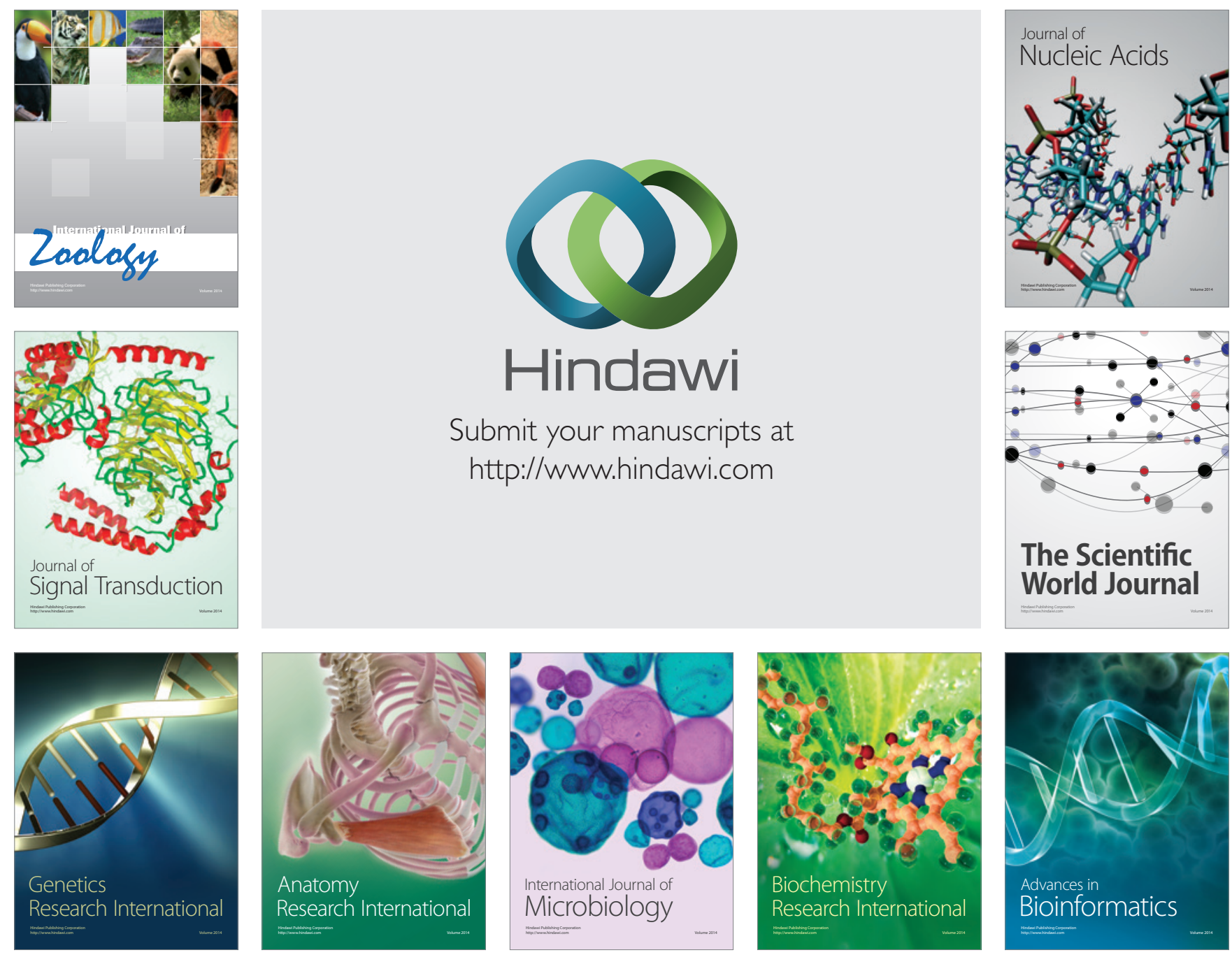

The Scientific World Journal
